\title{
Graphene and Carbon Nanotubes Synergistically Improved the Thermal Conductivity of Phenolic Resin
}

\author{
Han Wang \\ Sch Power \& Mech Engn Department, Wuhan University, Wuhan 430072, Hubei Province, Peoples R China \\ Corresponding Email: 2530707676@qq.com
}

\begin{abstract}
People discover the synergistic effect of graphene and carbon nanotubes on heat conduction in graphene carbon nanotubes / epoxy resin hybrid composites. In this article we added them into the phenolic resin and test the thermal conductivity. We found the thermal conductivity was increased by $6.5 \%$ in the phenolic resin by adding $0.45 \mathrm{wt} \%$ graphene and $0.15 \mathrm{wt} \%$ single wall carbon nanotubes (maintain the mass ratio $3: 1$ ). So if graphene and carbon nanotubes are added in proportion, thermal conductivity of phenolic resin will be improved significantly which is better than only carbon nanotubes or graphene.
\end{abstract}

\section{Introduction}

Graphene and carbon nanotubes are good conductors for electricity and heat because of their special structure. Now there are many useful way to produce Graphene [1] and carbon nanotubes [3, 4]. They are also very suitable to produce resin composite materials. In particular, the structure of carbon nanotubes is similar to polymers, whose main component is carbon. Carbon nanotubes and polymer composite materials will form a very completely binding interface which will have excellent performance [2]. Carbon nanotubes are easy to agglomerate [5], we can use ultrasonic to solve the problem [6]. Recently, people discover the synergistic effect of graphene and carbon nanotubes on heat conduction in graphene carbon nanotubes / epoxy resin hybrid composites, which has provided a new idea for the study of high thermal conductivity polymer composites. Some researchers found that the graphene carbon nanotube / epoxy hybrid composites have higher thermal conductivity than the same content of graphene composites and carbon nanotube composites. In the case of no change in the interface, the thermal conductivity of graphene and carbon nanotubes can significantly improve the thermal conductivity of epoxy resin composites. So, we are curious about whether it will has the same effect if we add them into the phenolic resin? It needs experiments to verify.

Because the acting force between adjacent graphite layers is weak, it is extremely easy to separate it and make many slices. Many scientists found graphene has good thermal conductivity and added it into some materials to make composite materials, which can improve its performance. At present, there are three ways to produce graphene. First is called chemical stripping: the first step is the preparation of graphite oxide, and then use the thermal expansion reduction to obtain graphene. The second is synthesis method using micro molecular or macro molecule as presoma. Which has high quality and high purity. The third method is called catalytic growth method.

The thermal conductivity of carbon nanotubes bear on its special structure and size. The thermal conductivity of can reach that of the graphene base. Which has the best performance of the heat conducting in nature. Carbon nanotubes has very high aspect ratio, which make it be very perfect for reinforced composite material. The structure of carbon nanotubes is similar to polymers, whose main component is carbon. Carbon nanotubes and polymer composite materials will form a very completely binding interface which will have excellent performance. Recently, carbon nanotube nanocomposites have attracted wide attention, most of them are carbon nanotube polymer composites, also there are few about the metal and nanotube reinforced composites with ceramic matrix.

\section{Testing program}

\subsection{Preparation of materials}

The materials we used in the experiment are layered oxidized graphene, which can be dispersed in liquid. In order to improve the dispersion of layered graphene, the following experiments are made:

Weigh $0.2 \mathrm{~g}$ layered graphene, $20 \mathrm{ml}$ ethanol in a beaker. Add graphene into ethanol, and use ultrasonic cleaning apparatus oscillating for 1 hour, aiming to make them uniformly dispersed. 
The turbid liquid of the oxidized graphene is poured into the filtering device, and a vacuum is drawn to accelerate the separation of the ethanol. Then put the filter paper into the heat treatment furnace, adjust to $180^{\circ} \mathrm{C}$. Half an hour after the filter paper being completely, take it out from the heat treatment furnace. Scrape the graphene on the filter paper and pour it back to the small glass bottle. The results showed that this method can improve the dispersion of graphene.

The dispersion of carbon nanotubes in the application process is an important step in the modification of the composite materials and the fabrication of nano devices. The dispersion of carbon nanotubes directly affect the performance of materials, therefore, how to effectively disperse carbon nanotubes, has become an important step in the practical application. Mechanical dispersion method is a simple physical method, which mainly depends on mechanical energy like external shear force or impact force in the dispersion of carbon nanotubes. Such as ball-milling or mixing. Ball-milling reduces carbon nanotube clusters but also has some destroy to the structure. Some people try to use the low speed ball mill the multi-wall carbon nanotubes, he found that multi-wall carbon nanotube open the ports and the length becomes shorter. After further milling for $20 \mathrm{~h}$, carbon nanotubes have obvious agglomeration. The energy generated by the collision between ceramic balls and carbon nanotubes breaks the carbon nanotubes. It is said that by controlling the grinding time, a large number of appropriate length of carbon nanotubes can be produced. We adopt the method of ball milling.

\subsection{Laboratory apparatus}

For sintering mixed sample we use the spark plasma sintering system in Japan Sumitomo Coal Mining Corporation production (SPS), just like Figure 1. Control the temperature at 200 degrees Celsius, and the pressure under the condition of 15MPA, the diameter of graphite mold is $15 \mathrm{~mm}$, sintering for ten minutes. To test the thermal diffusivity we use the laser thermal conductivity meter LFA457. It is shown in Figure 2.

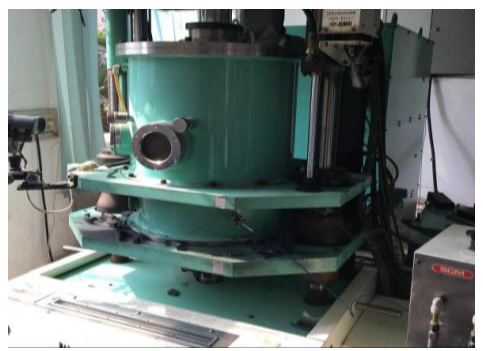

Fig. 1. SPS system.

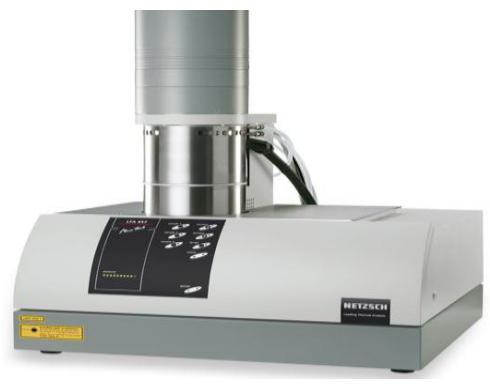

Fig. 2. Laser thermal conductivity meter.

\subsection{Preparation of composite materials}

The experimental scheme is as follows:

(1) Weigh 4 portion of powder (phenolic resin) with scales for $7 \mathrm{~g}$ each, grind them into uniform powder, adding to four beakers.

(2) Weigh $0.042 \mathrm{~g}$ carbon nanotubes (amount to $0.6 \mathrm{wt} \%$ of phenolic resin) into the No. 2 beaker. Weigh $0.042 \mathrm{~g}$ graphene (amount to $0.6 \mathrm{wt} \%$ of phenolic resin) into the No. 3 beaker. Weigh $0.0315 \mathrm{~g}$ graphene and $0.0105 \mathrm{~g}$ carbon nanotubes (mass ratio of graphene and carbon nanotubes is 3:1) into the No.4 beaker. The beakers were respectively mixed on mechanical grinding for an hour until evenly mixed, forming powder of uniform thickness.

(3) We used the spark plasma sintering system, and the diameter of graphite mold is $15 \mathrm{~mm}$ (the apparatus for testing thermal diffusivity requires the sample's diameter is $12 \sim 17 \mathrm{~mm}$, and the thickness is $2-3 \mathrm{~mm}$ ). Keep the temperature at 200 degrees Celsius, the pressure under the condition of $15 \mathrm{MPa}$, and sinter the sample. About ten minutes later we got the sintered samples. 


\subsection{Test of density $(\rho)$}

Polish the surface of samples with sandpaper of 200, making it a smooth surface, and measure the density by the way of drainage. We use the way of drainage to measure the volume of the sample, and measure the quality. We know that

$$
\rho=m / V
$$

We got the density of them just like Table 1 .

Table 1. Four groups of density.

\begin{tabular}{|l|c|c|c|c|}
\hline & Blank & CNTs & G & CNTs/G \\
\hline Volume[cm3] & 0.3 & 0.5 & 0.3 & 0.5 \\
\hline Quality[g] & 0.4215 & 0.6890 & 0.4165 & 0.6965 \\
\hline $\begin{array}{l}\text { density } \\
\text { [g/cm3] }\end{array}$ & 1.405 & 1.378 & 1.388 & 1.393 \\
\hline
\end{tabular}

\subsection{Calculation of specific heat capacity(C)}

We know that:

$$
Q=C \times M \times \Delta t
$$

And because our experiment is carried out at room temperature, the temperature is constant, the whole process of absorption of heat and heat release is balanced.

So we can have:

$$
C 1 \times m 1=C 2 \times m 2+C 3 \times m 3
$$

We can know the specific heat capacity:
(1) Blank group:
$1650 \mathrm{~J} /(\mathrm{kg} * \mathrm{~K})$.
(2) CNTs group:
$1648.5 \mathrm{~J} /(\mathrm{kg} * \mathrm{~K})$.
(3) G group:
$1649.1 \mathrm{~J} /(\mathrm{kg} * \mathrm{~K})$.
(4) $\mathrm{CNTs} / \mathrm{G}$ group: $1648.95 \mathrm{~J} /(\mathrm{kg} * \mathrm{~K})$.

\subsection{Calculation of thermal diffusivity (a)}

After testing the thermal diffusion coefficient we use the laser thermal conductivity meter LFA457. We can have the thermal diffusion coefficient shown as Table 2 .

Table 2. Thermal diffusivity (a) of four groups

\begin{tabular}{|c|l|l|l|l|}
\hline Property & \multicolumn{1}{|c|}{ Blank } & \multicolumn{1}{c|}{ CNT } & \multicolumn{1}{c|}{ G } & CNT/G \\
\hline Thickness[mm] & 1.666 & 2.633 & 1.500 & 2.820 \\
\hline Diameter[mm] & 15.10 & 15.00 & 15.00 & 15.10 \\
\hline $\mathrm{a} 3$ & 0.180 & 0.183 & 0.178 & 0.185 \\
\hline $\mathrm{a} 2$ & 0.168 & 0.181 & 0.179 & 0.187 \\
\hline $\mathrm{a} 3$ & 0.171 & 0.182 & 0.175 & 0.187 \\
\hline \begin{tabular}{c} 
average value \\
\hline
\end{tabular} & 0.173 & 0.182 & 0.177 & 0.186 \\
\hline
\end{tabular}

We can use: 


$$
\lambda(T)=a(T) \times C p(T) \times \rho(T) .
$$

Then the thermal conductivity is:
(1)Blank group:
$0.401 \mathrm{~W} /(\mathrm{m} * \mathrm{~K})$.
(2)CNTs group:
$0.413 \mathrm{~W} /(\mathrm{m} * \mathrm{~K})$.
(3) G group:
$0.405 \mathrm{~W} /(\mathrm{m} * \mathrm{~K})$.
(4)CNTs/G group:
$0.427 \mathrm{~W} /(\mathrm{m} * \mathrm{~K})$.

\section{Analysis}

\subsection{Synergistic heat conduction theory}

At present, there are more and more research on graphene and carbon nanotube composites. However, the mechanism of the graphene and carbon nanotubes co thermal conduction is not clear enough. There are many kinds of statements.

\subsubsection{Aninterconnected networks is produced}

Some people say that the causes synergistic thermal conductivity of graphene and carbon nanotubes are onedimensional carbon nanotubes (CNTs) and two-dimensional graphene (GNPs) cooperate with each other to form a crosslinked network, the network greatly reduces the thermal resistance, and they have the Percolation Effects [7]. Other people also support this view through the establishment of models and the use of statistical mechanics [8].

\subsubsection{Increase of the contact area}

Yang believes that the contact between carbon nanotubes is zero dimensional, and the contact between graphene and carbon nanotubes is one-dimensional [9]. The increase of the contact area produced a percolation effects, so it will produce the effect of synergistic heat conduction.

\subsubsection{Graphene and carbon nanotubes stimulate the percolation effect together.}

Chu established a theoretical model to describe the synergistic thermal conductivity of graphene and carbon nanotubes using the effective medium theory [10]. Through the analysis, they believe that the synergistic effect of graphene and carbon nanotubes may be due to the percolation effect of their co excitation.

\subsection{Analysis of the data}

Seeing the thermal conductivity from the experiment, we found the addition of graphene will increase the thermal conductivity, adding carbon nanotubes alone will make the thermal conductivity has a small increase, while adding graphene and carbon nanotubes can greatly improve the thermal conductivity. We can clearly verify the synergistic thermal conductivity of graphene and carbon nanotubes. With all kinds of conjecture mentioned above, they are all proposed reasonably but all have disadvantages, if we want to know what the real reason is, we need more experiments, observing the structure from the micro scale, and the establishment of a more practical models.

\section{Conclusions}

The thermal conductivity of the sample was improved by $2.9 \%$ in the phenolic resin by adding the single wall carbon nanotubes and the thermal conductivity of the sample was improved by $1.0 \%$ in the phenolic resin by adding. The thermal conductivity was increased by $6.5 \%$ in the phenolic resin by adding $0.45 \mathrm{wt} \%$ graphene and $0.15 \mathrm{wt} \%$ single wall carbon nanotubes (maintain the mass ratio 3:1). From above, we can see that if graphene and carbon nanotubes are added in proportion, thermal conductivity of phenolic resin will be improved significantly. And the effect is better than only adding carbon nanotubes or graphene.

\section{References}

1. A A Balandin: Nat Mater, 10(8): 569-81.(2011)

2. Z D Han, A Fina: Prog Polym Sci, 36(7): 914-44.

3. J R Potts, D R Dreyer, C W Bielawski, et al, Polymer, 52(1): 5-25(2011)

4. T Ramanathan, A A Abdala, S Stankovich, et al,Nat Nanotechnol, 3(6): 327-31. (2008)

5. A Yu, P Ramesh, X Sun, et al, Adv Mater, 20(24): 4740-4 (2008) 
6. S Y Yang, C C M Ma, C C Teng, et al, Carbon, 48(3): 592-603. (2010)

7. Y W Yi, X S Zeng, Materials Review,20:81-3.(2012)

8. R P Luo, East China University Of Science.(2013)

9. Y C Jia, K J Yu, K Qian, et al, Fiber Reinforced Plastics/Composites, 2:69-73.(2013)

10. K Chu, C C Jia, W S Li. Appl Phys Lett, 101(12): 211903. (2012) 